\title{
High-Accuracy $\mathrm{CO}_{2}$ Line Intensities Determined from Theory and Experiment
}

\author{
Oleg L. Polyansky, ${ }^{1,2}$ Katarzyna Bielska, ${ }^{3,4}$ Mélanie Ghysels, ${ }^{3}$ Lorenzo Lodi, ${ }^{1}$ Nikolai F. Zobov, ${ }^{2}$ \\ Joseph T. Hodges, ${ }^{3}$ and Jonathan Tennyson ${ }^{1, *}$ \\ ${ }^{1}$ Department of Physics and Astronomy, University College London, London WC1E 6BT, United Kingdom \\ ${ }^{2}$ Institute of Applied Physics, Russian Academy of Sciences, Ulyanov Street 46, Nizhny Novgorod 603950, Russia \\ ${ }^{3}$ Chemical Sciences Division, National Institute of Standards and Technology, Gaithersburg, Maryland 20899, USA \\ ${ }^{4}$ Institute of Physics, Faculty of Physics, Astronomy and Informatics, Nicolaus Copernicus University, \\ Grudziadzka 5, 87-100 Torun, Poland
}

(Received 14 February 2015; published 15 June 2015)

\begin{abstract}
Atmospheric $\mathrm{CO}_{2}$ concentrations are being closely monitored by remote sensing experiments which rely on knowing line intensities with an uncertainty of $0.5 \%$ or better. Most available laboratory measurements have uncertainties much larger than this. We report a joint experimental and theoretical study providing rotation-vibration line intensities with the required accuracy. The $a b$ initio calculations are extendible to all atmospherically important bands of $\mathrm{CO}_{2}$ and to its isotologues. As such, they will form the basis for detailed $\mathrm{CO}_{2}$ spectroscopic line lists for future studies.
\end{abstract}

DOI: 10.1103/PhysRevLett.114.243001

PACS numbers: 33.20.Ea, 33.20.Vq, 92.60.hg, 92.60.Vb

The quantity of carbon dioxide $\left(\mathrm{CO}_{2}\right)$ in Earth's atmosphere and its role in climate change has become a hotly debated topic both in scientific and nonscientific circles. Several agencies are flying (for example GOSAT [1], ACE [2], MIPAS [3], OCO-2 [4]) or preparing to launch (such as CarbonSat [5] and ASCENDS [6]) experiments or even whole missions to explicitly monitor the atmospheric $\mathrm{CO}_{2}$ content or to do this as part of wider scientific programs. Similarly, international ground-based networks such as the Total Carbon Column Observing Network (TCCON) [7] and the Network for the Detection of Atmospheric Composition Change [8] are also dedicated to monitoring atmospheric $\mathrm{CO}_{2}$. A major aim of this activity is to establish $\mathrm{CO}_{2}$ concentrations at the parts per million (ppm) level or, preferably, better. These projects aim not only to look at global $\mathrm{CO}_{2}$ levels and their variations, but also at sources and sinks of $\mathrm{CO}_{2}$. This activity is clearly vital to monitoring, and hopefully controlling, the anthropic greenhouse effect due to $\mathrm{CO}_{2}$ and, hence, climate change.

All $\mathrm{CO}_{2}$ remote sensing activities, from both the ground and space, rely on monitoring $\mathrm{CO}_{2}$ vibration-rotation spectra. They are, therefore, heavily dependent on laboratory spectroscopy for reliable parameters which are essential for interpreting the atmospheric spectroscopic data. These parameters are of three types: line centers, line profiles, and line intensities. Line centers or positions are established to high accuracy in many laboratory high resolution spectroscopy studies and, in general, do not

Published by the American Physical Society under the terms of the Creative Commons Attribution 3.0 License. Further distribution of this work must maintain attribution to the author(s) and the published article's title, journal citation, and DOI. require significant improvement for studies of Earth's atmosphere. Line profiles are more difficult to determine, but significant progress on these has been made in recent years with, for example, the inclusion of line mixing in both the HITRAN database [9] and many retrieval models. Here, we specifically focus on the issue of determining accurate line intensities. We present first results from a newly developed experiment designed to measure line intensities with an uncertainty of $0.3 \%$ and a new ab initio model also designed to achieve this level of accuracy.

Accurate line intensities are crucial for a successful retrieval since they relate directly to the $\mathrm{CO}_{2}$ column being retrieved. Without high accuracy values for line intensities, reliable retrievals are simply not possible. If current missions are to fulfil their goals, intensities accurate to $0.3 \%-0.5 \%$ are really required [10]. The laboratory procedures used up to now simply do not give this level of accuracy, and current retrievals values are limited by the available laboratory data [11-13]. Data are required not only for the main isotopologue, ${ }^{12} \mathrm{C}^{16} \mathrm{O}_{2}$, but also for isotopically substituted species such as ${ }^{14} \mathrm{C}^{16} \mathrm{O}_{2}$, which can be used to monitor recently added fossil-fuel-derived carbon emissions [14].

It is much harder to accurately measure line intensities than line positions in the laboratory. Typical uncertainties for experimental line intensity data used in atmospheric models and retrievals are $3 \%$ to $10 \%$ [15-17], and even high quality measurements (e.g., Boudjaadar et al. [18]) usually only provide accuracies in the $1 \%$ to $3 \%$ range, still significantly worse than required for precision remote sensing. There are three published studies aimed at measuring $\mathrm{CO}_{2}$ line intensities with an accuracy better than $1 \%[12,19,20]$. However, these studies only considered a small set of lines, in the case of Wuebbler et al. [12] only a single line, and do not agree with 
each other within theirgiven uncertainties. On the theory side, Huang et al. have recently performed a comprehensive treatment of the $\mathrm{CO}_{2}$ vibration-rotation spectrum [21-23] using theoretical procedures similar to those employed here. These studies produced an excellent spectroscopically determined potential energy surface (PES), which we use below, but had a more limited goal for the accuracy of their intensities of between $3 \%$ and $5 \%$.

The aim of this Letter is to provide an accurate theoretical solution to the problem of $\mathrm{CO}_{2}$ line intensities based on the application of high-accuracy, $a b$ initio quantum mechanical calculations tested against laboratory measurements of unprecedented low uncertainty. An advantage of our calculations is that they can be applied not only to all bands of importance, but also to all isotopically substituted variants of the molecule with essentially uniform precision. The disadvantage of ab initio methods has traditionally been that they are hard to perform to high accuracy, and it is difficult to estimate their uncertainty. Here, we present a joint experimental and theoretical study demonstrating an ab initio theoretical model capable of reproducing line intensities of ${ }^{12} \mathrm{C}^{16} \mathrm{O}_{2}$ with a combined uncertainty of about $0.3 \%$.

Theoretically, the intensity of a given spectral line is directly proportional to the square of the transition dipole, $\left(\mu_{i f}=\int \Psi_{i} \mu \Psi_{f} d \tau\right)$, where $\Psi_{i}$ and $\Psi_{f}$ are the initial and final wave function. For a vibration-rotation transition, $\underline{\mu}$ is the dipole moment surface (DMS) and the integration runs over all coordinates of the nuclei. The accurate calculation of molecular line intensities requires an accurate DMS and an accurate PES to provide wave functions. The rotationvibration Schrödinger equation is solved numerically to compute wave functions; we use the DVR3D program suite [24] for that purpose. Experience shows that best results are obtained by combining a spectroscopically determined PES with a fully $a b$ initio DMS [25].

Here, we use the highly accurate, empirical, $\mathrm{CO}_{2} \mathrm{PES}$ of Huang et al. [21]. What we require is an extrahigh-accuracy $\mathrm{CO}_{2}$ DMS. Systematic studies of the DMS of water $[25,26]$ have shown that sub-1\% accuracy requires consideration of many effects neglected in standard $a b$ initio treatments, and that there is a strong correlation between the accuracy of the DMS and the underlying PES associated with it [25]. A number of studies $[21,27]$ have also demonstrated the importance of generating the DMS using a dense grid of points. As shown for water [28,29], the key ingredients for a high-accuracy $a b$ initio treatment involve the use of multireference configuration interaction (MRCI) calculations with large basis sets (five- or six-zeta quality) and of large active spaces. It is, furthermore, necessary to add various corrections due to relativity (and even quantum electrodynamics) and failure of the Born-Oppenheimer (BO) approximation.

Water is a ten-electron system which lends itself to large systematic calculations. These calculations scale combinatorially and, therefore, are not currently computationally feasible for the 22-electron $\mathrm{CO}_{2}$ molecule. For this reason, it was necessary to design a new model: preliminary test calculations with this model, detailed in the Supplemental Material [30], were performed for CO.

Our calculations used the MOLPRO [38] quantum chemistry package to calculate the PES and DMS of $\mathrm{CO}_{2}$ at about 2000 randomly selected points with energies up to about $15000 \mathrm{~cm}^{-1}$ above the minimum. The calculations used all-electron MRCI and the aug-cc-pwCVQZ basis set. Relativistic corrections were determined from the oneelectron mass-velocity-Darwin (MVD1) term and fitted separately. The DMS was determined using finite-field effects rather than as an expectation value. As $\mathrm{CO}_{2}$ is heavier and more rigid than water, it transpired that non-BO corrections are of lesser importance, as has been shown before [21], and were not included. Full details of the calculation, including analytic representations of our final $a b$ initio DMS, which were obtained as polynomial expansions in symmetry coordinates, and the associated PES are given in the Supplemental Material [30].

DVR3D calculations for the three fundamental bands of ${ }^{12} \mathrm{C}^{16} \mathrm{O}_{2}$ using our ab initio PES show that the discrepancy between calculated and observed energy levels is about $1 \mathrm{~cm}^{-1}$, almost an order of magnitude smaller than the best previous $a b$ initio calculations [21]. This level of accuracy for the energy levels should be a pointer towards the accuracy of the corresponding DMS and suggests that the intensity of strong and medium lines should be predicted to within $0.5 \%$.

We made the most accurate measurements ever reported for $\mathrm{CO}_{2}$ line intensities using the frequency-stabilized cavity ring-down spectroscopy (FS-CRDS) [39-41] technique. FS-CRDS is a high-accuracy method that yields absorption spectra in terms of known integer multiples of the longitudinal mode spacing of a resonant optical cavity ( $x$ axis) and observed cavity decay times ( $y$ axis). In contrast to other absorption spectroscopy methods, this approach is immune to intensity and frequency fluctuations in the probe laser, consists of a relatively compact sample volume, and does not require explicit determination of the absorption path length. Our gas samples consisted of a known molar fraction of $\mathrm{CO}_{2}$ in air with values that were referenced to gravimetrically prepared primary standard mixtures. Further, to mitigate exchange of $\mathrm{CO}_{2}$ with internal surfaces of the spectrometer, the sample gas was continuously introduced into the absorption spectrometer. Using this approach, we measured the spectroscopic areas of 27 vibration-rotation transitions of $\mathrm{CO}_{2}$ in the wave number region $6200-6258 \mathrm{~cm}^{-1}$. These spectroscopic features correspond to $P$-and $R$-branch transitions of the ${ }^{12} \mathrm{C}^{16} \mathrm{O}_{2}$ (30 013)-(00 001) vibrational band.

The individual transitions were probed using the frequency-agile, rapid scanning spectroscopy method [42] to rapidly and precisely tune the laser in a stepwise fashion through successive cavity resonances. To this end, we used 
a high-finesse $\left(\sim 1.6 \times 10^{5}\right), 75$-cm-long cavity ring-down spectrometer whose length was actively stabilized with respect to a frequency-stabilized $\mathrm{HeNe}$ laser having a drift (on the time scale of spectrum acquisition) of less than $0.5 \mathrm{MHz}$. Two continuous-wave, distributed-feedback diode lasers $(1 \mathrm{MHz}$ nominal line width) provided the wavelength coverage required to interrogate all the $\mathrm{CO}_{2}$ transitions reported.

Relative standard deviations in the absorption coefficient, $\alpha_{\text {tot }}$, at a given frequency, $\nu$, were $0.08 \%$, and over the entire $10 \mathrm{GHz}$ spectral window, spectra were acquired in $\sim 45 \mathrm{~s}$ giving spectrum signal-to-noise ratios of $\sim 5000: 1$. Between 20 and 100 spectra were acquired at pressures corresponding to $6.7,13.3$, and $20 \mathrm{kPa}$. Typically, we fit each observed line with the sum of a linear baseline and multiple quadratic speed-dependent Nelkin-Ghatak profiles [43] (one for each observed line) to the etalon-subtracted spectrum $\left[\alpha_{\text {tot }}(\nu)\right]$. This analysis gave the fitted area $A(p, T)$ at each pressure, $p$, and temperature, $T$. Here, we report the average, measured, pressure-independent line intensity $S$.

Gas pressure and cell temperature were actively stabilized to minimize drift during the acquisition of each spectrum, and the measurements of $p$ and $T$ were traceable to National Institute of Standards and Technology primary standards. Nominal relative standard uncertainties (RSUs) considered include: spectrum tuning step size $(0.001 \%)$, statistical uncertainty in fitted area $(0.01 \%)$, pressure $(0.015 \%)$, systematic residual area uncertainty $(0.02 \%)$, uncertainty in isotopic composition $(0.02 \%)$, gas temperature $(0.05 \%)$, variations in baseline etalons $(0.05 \%-0.9 \%)$, and sample molar fraction (0.07\%). Adding these components in quadrature gives RSU values ranging from $0.1 \%-1 \%$, with the strongest lines being relatively insensitive to baseline uncertainties and having RSUs between $0.1 \%$ and $0.2 \%$. We attribute these exceptionally low combined uncertainties to the high fidelity and sensitivity of the FS-CRDS method and to the accurately known $\mathrm{CO}_{2}$ concentration in the sample gas.

Figure 1 compares literature values for the (30013)(00001) band of ${ }^{12} \mathrm{C}^{16} \mathrm{O}_{2}$ with the present $a b$ initio calculations and our measurements. These results are summarized as the relative intensity difference versus rotational quantum number $m$. The literature results include two independent sets of measurements reported by Boudjaadar et al. [18] (LPPM and GSMA) and other intensities given in HITRAN 2012 [9]. These three data sets are based on Fourier transform spectroscopy measurements of pure carbon dioxide samples. We also include intensities archived in the carbon dioxide spectral data bank (CDSD) [44], which are based on fits to several spectroscopic measurements. When taken together, the relative intensity differences have a standard deviation and mean of $0.9 \%$, and $0.03 \%$, respectively. We find the smallest root-mean-square deviation $(0.23 \%)$ and minimum absolute relative difference $(0.33 \%)$ when comparing the present calculations to our

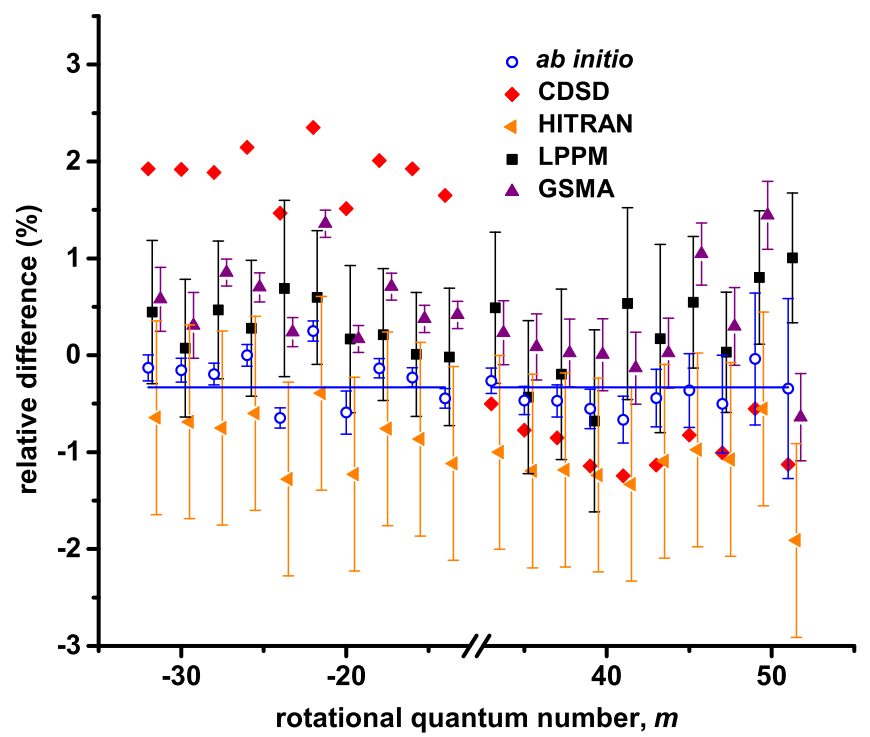

FIG. 1 (color online). Ab initio intensities (this work) and literature values relative to the present measurements versus the rotational quantum number $m$. Error bars represent \pm 1 standard deviation. The average, weighted (based on the inverse of the $\mathrm{RSU}^{2}$ ) relative difference between the calculations and the present measured values equals- $0.33 \%$ (indicated by the horizontal line) and has a standard error of $0.05 \%$. This comparison gives a root-mean-square deviation of $0.23 \%$ consistent with the measurement RSU. Data sources are CDSD [44], HITRAN [9], LPPM [18], and GSMA [18].

measurements. These results confirm that the relative uncertainties of the present $a b$ initio intensity calculations and measurements are in good agreement and well below the percent level, which constitutes a substantial improvement over previous intensity measurements.

Intensities of the (20012)-(00001) band of ${ }^{12} \mathrm{C}^{16} \mathrm{O}_{2}$ have also been the subject of precision studies. As shown in Fig. 2, only one of the intensities measured by Casa et al.

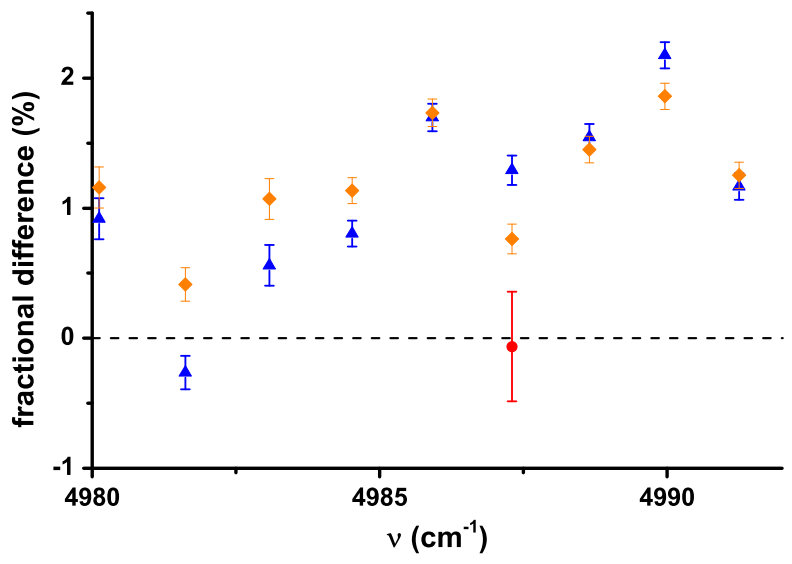

FIG. 2 (color online). Ab initio line intensities (this work) for the (20012)-(00001) band of the ${ }^{12} \mathrm{C}^{16} \mathrm{O}_{2}$ molecule at $296 \mathrm{~K}$ compared to the measurements of Casa et al. in 2007 [19] (blue triangles) and 2009 [20] (orange diamonds), and the single 2011 measurement of Wuebbeler et al. [12] (red circle). 
TABLE I. Line intensities, in $\mathrm{cm} /$ molecule, of the $R(10)$ transitions of the main bands of ${ }^{12} \mathrm{C}^{16} \mathrm{O}_{2}$ at $296 \mathrm{~K}$. Our calculations $(\mathrm{C})$ versus HITRAN $(\mathrm{H})$ [9]; "Uncertainty" gives the stated HITRAN uncertainty for each line. (Powers of 10 are in parentheses).

\begin{tabular}{|c|c|c|c|c|c|}
\hline Band & $\tilde{\nu} / \mathrm{cm}^{-1}$ & $I(\mathrm{H})$ & $I(\mathrm{C})$ & $(\mathrm{H}-\mathrm{C}) / \mathrm{H}(\%)$ & Uncertainty $(\%)$ \\
\hline (01101) & 676.01 & $1.524(-19)$ & $1.519(-19)$ & 0.35 & 5 \\
\hline (11102) & 1941.12 & $7.262(-25)$ & $5.975(-25)$ & 17.73 & 20 \\
\hline (11101) & 2085.46 & $2.576(-23)$ & $2.571(-23)$ & 0.19 & 10 \\
\hline (00011) & 2357.32 & $3.116(-18)$ & $3.117(-18)$ & -0.04 & 5 \\
\hline (21103) & 3190.15 & $1.302(-25)$ & $1.284(-25)$ & 1.42 & 20 \\
\hline (21102) & 3347.91 & $1.694(-24)$ & $1.721(-24)$ & -1.61 & 20 \\
\hline (21101) & 3509.27 & $1.071(-24)$ & $1.108(-24)$ & -3.47 & 20 \\
\hline (10012) & 3621.06 & $3.351(-20)$ & $3.395(-20)$ & -1.31 & 5 \\
\hline (02211) & 3667.64 & $1.765(-25)$ & $1.787(-25)$ & -1.23 & 20 \\
\hline (10011) & 3722.94 & $5.162(-20)$ & $5.228(-20)$ & -1.27 & 5 \\
\hline (31104) & 4424.87 & $3.062(-27)$ & $3.356(-27)$ & -9.61 & 20 \\
\hline (31103) & 4599.65 & $3.865(-26)$ & $3.986(-26)$ & -3.14 & 20 \\
\hline (31102) & 4761.96 & $5.544(-26)$ & $5.687(-26)$ & -2.58 & 20 \\
\hline (20013) & 4861.93 & $2.370(-22)$ & $2.370(-22)$ & 0.00 & 5 \\
\hline (12212) & 4896.40 & $4.070(-27)$ & $3.972(-27)$ & 2.40 & 20 \\
\hline (31101) & 4946.98 & $3.061(-27)$ & $2.362(-27)$ & 22.85 & 20 \\
\hline (20012) & 4985.93 & $1.127(-21)$ & $1.156(-21)$ & -2.60 & 5 \\
\hline (12211) & 5070.13 & $4.949(-27)$ & $4.889(-27)$ & 1.21 & 20 \\
\hline (30013) & 6236.03 & $1.515(-23)$ & $1.551(-23)$ & -2.38 & 10 \\
\hline
\end{tabular}

$[19,20]$ is within $0.3 \%$ of our calculations, while the average relative difference is greater than $1 \%$. However, for one of these problematic lines, $R(12)$, the intensity has been remeasured independently by Wuebbeler et al. [12]. This remeasured value is within $0.2 \%$ of our calculated value. It would appear that the intensities of Casa et al. are measured less accurately than claimed.

Table 1 compares our calculated intensities with those given in HITRAN for different $R(10)$ transitions: these results are typical of comparisons with other transitions. Good agreement, within the rather large HITRAN uncertainties, is found in all cases. In particular, we note that very good agreement, to about $0.4 \%$ and $0.2 \%$, respectively, is obtained for transitions within the two fundamental bands, (01101)-(00001) and (00011)-(00001) (fuller results are given in the Supplemental Material [30]). We suggest that this is not a coincidence. These HITRAN intensities are the result of calculations using a fitted, effective DMS [45]. As the constants of the effective DMS responsible for these strong fundamental bands are determined by fits to both these and over 100 other bands, the large amount of data available from a wide variety of experiments leads to an overall accuracy of the line intensities which should significantly improve, on the average, 2\%-5\% uncertainty in the experimental data used in the fit. The excellent agreement demonstrates both the extreme accuracy of our calculations and the effectiveness of the Hamiltonian fit in this case. Furthermore, for all bands, the relative differences between the HITRAN and calculated intensities are generally much smaller in magnitude than the rather large HITRAN uncertainties given in Table 1. We suggest that our new intensities generally represent a considerable improvement in accuracy and that the HITRAN uncertainties may be reduced substantially by taking into account the present calculations.

We present new high accuracy measurements and $a b$ initio calculations of transition intensities for the key ${ }^{12} \mathrm{C}^{16} \mathrm{O}_{2}$ molecule. Agreement between calculations and experiments is at the $0.3 \%$ level, which represents a significant improvement over previous theoretical and measured values. Our theoretical procedure is capable of producing comprehensive line lists not only for ${ }^{12} \mathrm{C}^{16} \mathrm{O}_{2}$, but also for the various isotopically substituted versions. These line lists, for which experimental line frequencies can be used, will give a significant and important improvement in $\mathrm{CO}_{2}$ line intensities available for atmospheric remote sensing and other studies. These line lists will be presented elsewhere.

This work was supported by UK Natural Environment Research Council under Grant No. NE/J010316, ERC Advanced Investigator Project No. 267219, the Russian Fund for Fundamental Studies, and the Climate Sciences Measurements Program of the National Institute of Standards and Technology and State Project IAP RAS No. 0035-2014-009.

*j.tennyson@ucl.ac.uk.

[1] A. Butz, S. Guerlet, O. Hasekamp, D. Schepers, A. Galli, I. Aben, C. Frankenberg, J. M. Hartmann, H. Tran, A. Kuze et al., Geophys. Res. Lett. 38, L14812, (2011).

[2] P. F. Bernath, C. T. McElroy, M. C. Abrams, C. D. Boone, M. Butler, C. Camy-Peyret, M. Carleer, C. Clerbaux, 
P. F. Coheur, R. Colin et al., Geophys. Res. Lett. 32, L15S01 (2005).

[3] H. Fischer, M. Birk, C. Blom, B. Carli, M. Carlotti, T. von Clarmann, L. Delbouille, A. Dudhia, D. Ehhalt, M. Endemann et al., Atmos. Chem. Phys. 8, 2151 (2008).

[4] D. Crisp, R. Atlas, F. Breon, L. Brown, J. Burrows, P. Ciais, B. Connor, S. Doney, I. Fung, D. Jacob et al., Adv. Space Res. 34, 700 (2004).

[5] V. A. Velazco, M. Buchwitz, H. Bovensmann, M. Reuter, O. Schneising, J. Heymann, T. Krings, K. Gerilowski, and J. P. Burrows, Atmos. Meas. Tech. 4, 2809 (2011).

[6] J. B. Abshire, H. Riris, G. R. Allan, C. J. Weaver, J. Mao, X. Sun, W. E. Hasselbrack, A. Yu, A. Amediek, Y. Choi et al., Proc. SPIE Int. Soc. Opt. Eng. 7832, 78320D (2010).

[7] D. Wunch, G. C. Toon, J.-F. L. Blavier, R. A. Washenfelder, J. Notholt, B. J. Connor, D. W. T. Griffith, V. Sherlock, and P. O. Wennberg, Phil. Trans. R. Soc. A 369, 2087 (2011).

[8] F. Hase, Atmos. Meas. Tech. 5, 603 (2012).

[9] L. S. Rothman, I. E. Gordon, Y. Babikov, A. Barbe, D. C. Benner, P. F. Bernath, M. Birk, L. Bizzocchi, V. Boudon, L. R. Brown et al., J. Quant. Spectrosc. Radiat. Transfer 130, 4 (2013).

[10] C. E. Miller, D. Crisp, P. L. DeCola, S. C. Olsen, J. T. Randerson, A. M. Michalak, A. Alkhaled, P. Rayner, D. J. Jacob, P. Suntharalingam et al., J. Geophys. Res. 112, D10314 (2007).

[11] B. J. Connor, H. Boesch, G. Toon, B. Sen, C. Miller, and D. Crisp, J. Geophys. Res. 113, D05305, (2008).

[12] G. Wuebbeler, G. J. P. Viquez, K. Jousten, O. Werhahn, and C. Elster, J. Chem. Phys. 135, 204304 (2011).

[13] C. E. Sioris, C. D. Boone, R. Nassar, K. J. Sutton, I. E. Gordon, K. A. Walker, and P. F. Bernath, Atmos. Meas. Tech. 7, 2243 (2014).

[14] S. J. Lehman, J. B. Miller, C. Wolak, J. Southon, P. P. Tans, S. A. Montzka, C. Sweeney, A. Andrews, B. LaFranchi, T. P. Guilderson et al., Radiocarbon 55, 1484 (2013).

[15] L. Wang, V. I. Perevalov, S. A. Tashkun, Y. Ding, and S.-M. Hu, J. Mol. Spectrosc. 234, 84 (2005).

[16] B. V. Perevalov, A. Campargue, B. Gao, S. Kassi, S. A. Tashkun, and V. I. Perevalov, J. Mol. Spectrosc. 252, 190 (2008).

[17] K. F. Song, S. Kassi, S. A. Tashkun, V. I. Perevalov, and A. Campargue, J. Quant. Spectrosc. Radiat. Transfer 111, 332 (2010).

[18] D. Boudjaadar, J.-Y. Mandin, V. Dana, N. Picqué, and G. Guelachvili, J. Mol. Spectrosc. 236, 158 (2006).

[19] G. Casa, D. A. Parretta, A. Castrillo, R. Wehr, and L. Gianfrani, J. Chem. Phys. 127, 084311 (2007).

[20] G. Casa, R. Wehr, A. Castrillo, E. Fasci, and L. Gianfrani, J. Chem. Phys. 130, 184306 (2009).

[21] X. Huang, D. W. Schwenke, S. A. Tashkun, and T. J. Lee, J. Chem. Phys. 136, 124311 (2012).

[22] X. Huang, R. S. Freedman, S. A. Tashkun, D. W. Schwenke, and T. J. Lee, J. Quant. Spectrosc. Radiat. Transfer 130, 134 (2013).
[23] X. Huang, R. R. Gamache, R. S. Freedman, D. W. Schwenke, and T. J. Lee, J. Quant. Spectrosc. Radiat. Transfer 147, 134 (2014).

[24] J. Tennyson, M. A. Kostin, P. Barletta, G. J. Harris, O. L. Polyansky, J. Ramanlal, and N. F. Zobov, Comput. Phys. Commun. 163, 85 (2004).

[25] L. Lodi, J. Tennyson, and O. L. Polyansky, J. Chem. Phys. 135, 034113 (2011).

[26] L. Lodi, R. N. Tolchenov, J. Tennyson, A. E. Lynas-Gray, S. V. Shirin, N. F. Zobov, O. L. Polyansky, A. G. Császár, J. van Stralen, and L. Visscher, J. Chem. Phys. 128, 044304 (2008).

[27] M. Pavanello, L. Adamowicz, A. Alijah, N. F. Zobov, I. I. Mizus, O. L. Polyansky, J. Tennyson, T. Szidarovszky, A. G. Császár, M. Berg et al., Phys. Rev. Lett. 108, 023002 (2012).

[28] O. L. Polyansky, A. G. Császár, S. V. Shirin, N. F. Zobov, P. Barletta, J. Tennyson, D. W. Schwenke, and P. J. Knowles, Science 299, 539 (2003).

[29] O. L. Polyansky, R. I. Ovsyannikov, A. A. Kyuberis, L. Lodi, J. Tennyson, and N. F. Zobov, J. Phys. Chem. A 117, 9633 (2013).

[30] See Supplemental Material at http://link.aps.org/ supplemental/10.1103/PhysRevLett.114.243001 which includes Refs. [31-37], for a detailed description of the calculations and experiments, and tabulations of the calculated and measured line intensities.

[31] L. Lodi and J. Tennyson, J. Phys. B 43, 133001 (2010).

[32] P. Pyykkö, K. G. Dyall, A. G. Császár, G. Tarczay, O. L. Polyansky, and J. Tennyson, Phys. Rev. A 63, 024502 (2001).

[33] O. L. Polyansky, L. Lodi, J. Tennyson, and N. F. Zobov (to be published).

[34] J. Tennyson and B. T. Sutcliffe, J. Mol. Spectrosc. 101, 71 (1983).

[35] R. M. Verkouteren, Anal. Chem. 71, 4740 (1999).

[36] D. A. Long, S. Wójtewicz, and J. T. Hodges, Proc. SPIE Int. Soc. Opt. Eng. 8726, 872600 (2013).

[37] J. Courtois, K. Bielska, and J. T. Hodges, J. Opt. Soc. Am. B 30, 1486 (2013).

[38] H.-J. Werner, P. J. Knowles, G. Knizia, F. R. Manby, and M. Schütz, Wiley Interdiscip. Rev.: Comput. Mol. Sci. 2, 242 (2012).

[39] J. T. Hodges, H. P. Layer, W. M. Miller, and G. E. Scace, Rev. Sci. Instrum. 75, 849 (2004).

[40] J. T. Hodges and R. Ciuryło, Rev. Sci. Instrum. 76, 023112 (2005).

[41] D. A. Long, A. Cygan, R. D. van Zee, M. Okumura, C. E. Miller, D. Lisak, and J. T. Hodges, Chem. Phys. Lett. 536, 1 (2012).

[42] G. W. Truong, K. O. Douglass, S. E. Maxwell, R. D. van Zee, D. F. Plusquellic, J. T. Hodges, and D. A. Long, Nat. Photonics 7, 532 (2013).

[43] R. Ciuryło, Phys. Rev. A 58, 1029 (1998).

[44] S. A. Tashkun and V.I. Perevalov, J. Quant. Spectrosc. Radiat. Transfer 112, 1403 (2011).

[45] S. A. Tashkun, V. I. Perevalov, J.L. Teffo, and V.G. Tyuterev, J. Quant. Spectrosc. Radiat. Transfer 62, 571 (1999). 